\title{
Variations in Axillary Staging: Much Ado About Nothing?
}

\author{
Tari A. King, MD, FACS \\ Memorial Sloan-Kettering Cancer Center, New York, NY 10065
}

The debate regarding the role of lymphadenectomy in breast cancer persists despite the availability of 25-year follow-up data from the National Surgical Adjuvant Breast and Bowel Project (NSABP) B04 trial comparing radical mastectomy, total mastectomy with regional nodal irradiation, and total mastectomy with no nodal treatment, a study that was designed to address this question. ${ }^{1}$ This trial failed to show a survival benefit attributable to regional nodal surgery or radiation; however, critical review revealed that the trial was likely too underpowered to show this difference, and axillary lymph node dissection (ALND) remained an important component of surgical therapy to accurately stage the axilla, guide systemic therapy decisions, and minimize regional recurrence. ${ }^{2}$ Sentinel lymph node (SLN) biopsy, an accurate and less invasive alternative to axillary node dissection, has become the new standard of care for axillary staging, and adoption of this technique has allowed surgeons to avoid axillary dissection and many of its attendant morbidities in node-negative patients. Questions still remain, however, as to whether ALND is always necessary in patients with positive SLNs and considerable effort has gone into the development of nomograms and predictive models to identify a subset of patients with a sufficiently low probability of having additional non-SLN metastases for whom completion node dissection may be avoided.

With the early closure of the American College of Surgeons Oncology Group (ACOSOG) Z0011 trial, it is unlikely that we will have ever have definitive evidence as to whether ALND improves survival in patients with positive lymph nodes; yet axillary lymph node staging remains an integral component of breast cancer treatment, and axillary lymph node status remains the most significant prognostic factor for disease recurrence and survival. All current

(C) Society of Surgical Oncology 2008

First Received: 21 October 2008;

Published Online: 18 December 2008

T. A. King, MD, FACS

e-mail: Kingt@mskcc.org guidelines for the management of invasive breast cancer include the performance of an axillary staging procedure to guide further treatment decisions, and the National Comprehensive Cancer Network ( $\mathrm{NCCN}$ ) and the American Society of Clinical Oncology state that SLN biopsy (SLNB) is the preferred method of axillary sampling when available. ${ }^{3,4} \mathrm{NCCN}$ guidelines further state that the performance of ALND is considered optional in patients with "particularly favorable tumors, patients for whom the selection of adjuvant systemic therapy is likely to be unaffected, for the elderly, and for those with serious comorbid conditions." These caveats allow physicians to balance the risks and the benefits of the procedure for an individual patient, i.e., the art of medicine, but should not result in lesser-quality care for any particular group of patients.

In this issue, Halpern et al. have found that significant variation exists in the performance of axillary staging procedures with respect to age, race, and socioeconomic class. ${ }^{5}$ Although there is a growing body of literature citing disparities in breast cancer care across race and socioeconomic class, ${ }^{6}$ the most significant finding in this analysis of the National Cancer Database (NCDB) is the omission of lymph node biopsy (LNB) in the older population. Patients in the oldest quartile (age $\geq 73$ ) were more than three times as likely not to receive an axillary staging procedure compared to their younger counterparts (age $<51$ years). Such variation in axillary surgery in the elderly should not come as a surprise. In the era before SLNB (1985-1995), a NCDB report identified that women older than 70 were more than twice as likely not to receive ALND when undergoing breast-conserving surgery (BCS) for early-stage breast cancer, ${ }^{7}$ and after the introduction of SLNB (1998-2005), the authors of the current paper previously reported that among all patients undergoing axillary surgery for earlystage breast cancer, older patients were less likely to receive SLNB (odds ratio, 0.8; 95\% confidence interval, 0.780.82). ${ }^{8}$ Halpern et al. also cite other published reports of variations in the surgical management of breast cancer for 
the elderly. The current findings are therefore not new, but they are the most recent data available from the NCDB (2003-2005) and they include any means of axillary staging: unspecified LNB/aspiration, SLNB only, ALND only, or a combination of SLNB and ALND.

The basis for omitting axillary surgery in the elderly is likely multifactorial. Some would argue that the morbidity of axillary dissection and the risk associated with general anesthesia outweighs the benefit in clinically node-negative older women, or that axillary staging information will not change adjuvant therapy recommendations, which are often limited to hormonal therapy in this group. Although the ability to perform SLNB minimizes the morbidity of ALND, even this procedure is not without risk of complication, as highlighted by the ACOSOG Z0010-11 trial, where $8.6 \%$ of women experienced axillary paresthesias, $3.8 \%$ experienced decreased abduction of the upper extremity, and $7 \%$ of women developed lymphedema. ${ }^{9}$ The technical expertise and technology infrastructure required for SLNB was also slow to reach many low-volume community hospitals. All of these arguments appear to be reflected in the data from the NCDB where a higher proportion of women who did not receive LNB had smaller tumors, underwent BCS as opposed to mastectomy, and were treated in low-volume and community hospitals where older women often receive their care. Further, the increasing rate of LNB in 2004 and 2005 compared with 2003 supports the assumption that SLNB likely became available at more centers over the time period of this study. It is impossible to determine the impact of significant medical comorbidities or to understand factors associated with patient-provider communication from such population-based studies, but certainly these factors must also be considered when analyzing patterns of care in the elderly.

Accepting the findings and the limitations of this study, we are left with the question: does the omission of LNB among the elderly represent poor care or good clinical judgment? Surveillance Epidemiology and End Results data demonstrate that approximately $70 \%$ of breast cancer patients are at least 60 years old and $46 \%$ are age 70 and older, yet aging is a highly individualized process, and chronological age may or may not be an accurate assessment of individual life expectancy. ${ }^{10}$ Competing sources of death or comorbidities are important considerations when assessing the risks and benefits of any therapy in elderly breast cancer patients, including surgical staging procedures. Aging is also associated with a higher prevalence of hormone receptor-positive, well-differentiated tumors, with lower production of angiogenic growth factors, lower proliferation rate, and a lesser degree of lymph node involvement, suggesting that in general, breast cancer is less aggressive in the elderly. ${ }^{11,12}$ Data from the 50-69year age group from the Early Breast Cancer Trialists
Collaborative Group Overview also demonstrate that for patients with hormone receptor-positive disease, endocrine therapy provides most of the advantage associated with adjuvant treatments, and no major safety issues have been identified in older patients in large clinical trials of the aromatase inhibitors. ${ }^{13,14}$ Therefore, if axillary surgery to determine node status is associated with increased risk and morbidity and is less relevant for adjuvant treatment decisions, what is the true value of axillary surgery in this population?

The International Breast Cancer Study Group (IBCSG) attempted to answer this question by randomizing patients 60 years of age and older to axillary clearance or no axillary surgery after either mastectomy or lumpectomy. ${ }^{15}$ All patients were clinically node negative and received tamoxifen. The trial opened in May 1993 with the primary endpoint of assessing disease-free survival between the two groups (axillary clearance vs. no axillary clearance); however, as a result of slow accrual, the study was redesigned in November 2000 to assess whether avoiding axillary clearance improved quality of life. The median age of patients enrolled $(n=473)$ was 74 years, $56 \%$ had T1 tumors, $45 \%$ underwent mastectomy, 33\% had BCS with radiotherapy, $23 \%$ had BCS without radiotherapy (equally represented in both groups), and $28 \%$ of patients undergoing axillary clearance had positive nodes. At a median follow-up of 6.6 years, there was no difference in diseasefree or overall survival between the two groups, irrespective of estrogen receptor status, and specifically there was no difference in the incidence of axillary recurrence between the two treatment options. Regional recurrence or reappearance of disease in the axilla was observed for only $2 \%$ of the patients overall (3\% without axillary clearance and $1 \%$ with axillary clearance). Avoiding axillary surgery was associated with improved quality-of-life scores during the first year of follow-up; however, these differences did not persist over time.

Acknowledging the small study size, the authors of the IBCSG trial performed a predictive power calculation and concluded that if the trial had fully accrued as originally designed, there would have been a less than $3 \%$ chance of observing a statistically significant benefit in disease-free survival for axillary clearance. This does not rule out the possibility that a small benefit may be observed with large numbers of patients, but this been difficult to show in metaanalyses including breast cancer patients of all ages and would likely be harder to show when limited to the elderly. Thus when the arguments for axillary surgery are weighed against the potential increased morbidity of these procedures in the elderly, the evidence does not support the value of routine axillary surgery to guide adjuvant treatment decisions, minimize regional recurrence, or improve survival in clinically node-negative patients. For older 
patients with clinically positive axillary nodes, the results of the IBCSG trial are also reassuring, demonstrating that for most women, their long-term daily functioning or quality of life will not be greatly altered by the performance of an axillary dissection.

Whether the importance of axillary surgery for staging will persist for breast cancer patients of any age in an era of genomic medicine-where the gene expression profile of the tumor may trump the prognostic value of nodal statusremains to be seen. For now, one cannot forget that the elderly population is quite heterogeneous with respect to comorbid conditions and degree of disability and likewise may vary in terms of degree of tolerance for therapy. In the absence of outcome data for the elderly patients represented in the current study, we cannot conclude that the omission of LNB was poor care. Instead, perhaps we should consider that Halpern et al. have used the NCDB to demonstrate that physicians taking care of elderly breast cancer patients are in fact acknowledging evidence-based practice guidelines and practicing the art of medicine.

\section{REFERENCES}

1. Fisher B, Jeong JH, Anderson S, et al. Twenty-five-year followup of a randomized trial comparing radical mastectomy, total mastectomy, and total mastectomy followed by irradiation. $N$ Engl J Med. 2002;347:567-75.

2. Harris JR, Osteen RT. Patients with early breast cancer benefit from effective axillary treatment. Breast Cancer Res Treat. 1985;5:17-21.

3. National Comprehensive Cancer Network. NCCN Practice Guidelines in Oncology: Breast Cancer. V.2.2008. Available at: http://www.nccn.org/professionals/physician_gls/PDF/breast.pdf. Accessed November 18, 2008.
4. Lyman GH, Giuliano AE, Somerfield MR, et al. American society of clinical oncology guideline recommendations for sentinel lymph node biopsy in early-stage breast cancer. J Clin Oncol. 2005;23:7703-20.

5. Halpern MT, Chen AY, Marlow NS, et al. Disparities in receipt of lymph node biopsy among early-stage female breast cancer patients. Ann Surg Oncol. DOI:10.1245/s10434-008-0205-7.

6. Newman LA, Martin IK. Disparities in breast cancer. Curr Probl Cancer. 2007;3:134-56.

7. Bland KI, Scott-Conner CE, Menck H, et al. Axillary dissection in breast-conserving surgery for stage I and II breast cancer: a national cancer data base study of patterns of omission and implications for survival. J Am Coll Surg. 1999;188:586-95.

8. Chen AY, Halpern MT, Schrag NM, et al. Disparities and trends in sentinel lymph node biopsy among early-stage breast cancer patients (1998-2005). J Natl Cancer Inst. 2008;100:462-74.

9. Wilke LG, McCall LM, Posther KE, et al. Surgical complications associated with sentinel lymph node biopsy: results from a prospective international cooperative group trial. Ann Surg Oncol. 2006;13:491-500.

10. Ries LAG, Melbert D, Krapcho M, et al. SEER cancer statistics review, 1975-2004. Bethesda, MD: National Cancer Institute.

11. Rao VS, Garimella V, Hwang M, et al. Management of early breast cancer in the elderly. Int J Cancer. 2007;120:1155-60.

12. Daidone MG, Coradini D, Martelli G, et al. Primary breast cancer in elderly women: biological profile and relation with clinical outcome. Crit Rev Oncol Hematol. 2003;45:313-25.

13. Early Breast Cancer Trialists' Collaborative Group (EBCTCG). Effects of chemotherapy and hormonal therapy for early breast cancer on recurrence and 15-year survival: an overview of the randomised trials. Lancet. 2005;365(9472):1687-717.

14. Balducci L. Treating elderly patients with hormone sensitive breast cancer: what do the data show? Cancer Treat Rev. 2008 (in press).

15. International Breast Cancer Study Group. Tamoxifen after adjuvant chemotherapy for premenopausal women with lymph nodepositive breast cancer: International breast cancer study group trial 13-93. J Clin Oncol. 2006;24:1332-41. 